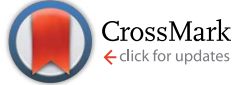

Cite this: J. Mater. Chem. B, 2014, 2, 7118

Received 3rd August 2014 Accepted 8th September 2014

DOI: $10.1039 / c 4 t b 01283 f$

\section{Phosphonate based organosilane modification of a simultaneously protein resistant and bioconjugable silica surface $\uparrow$}

\author{
Erol Ozgur,,$^{\mathrm{ab}}$ Pelin Torent $\stackrel{t}{\mathrm{ab}}$ and Mehmet Bayindir $^{\star a b c}$
}

www.rsc.org/MaterialsB

A facile method to coat silica surfaces with THPMP is introduced, forming simultaneously a protein resistant and bioconjugable surface. The coating is experimentally identified and its anti-fouling and bioconjugable characteristics are demonstrated.

Chemical modification of surfaces, which enables unprecedented manipulation of the characteristics of materials, can be attained by a virtually indefinite number of different compounds and methods. Regarding the accommodation of molecules over surfaces, self-assembly strategies depending on spontaneous interactions of the molecules with the surface for the formation of mono- or multilayers are among the most frequently utilized. Self-assembly generally occurs via functional head groups having high affinity towards the substrate, anchoring the molecule covalently or non-covalently to the surface. Among various different head groups, silanes are particularly important and widely used, while the knowledge regarding their assembly is far more empirical, besides being practical. Silanes are assumed to form monolayers over the surfaces; yet this is a controversial issue since their assembly depends highly on the environmental conditions, obstructing the formation of films with well-defined characteristics. Also polymerization frequently occurs during their assembly. ${ }^{1}$

The characteristics of a silane based coating depend on many factors such as density, ${ }^{2}$ chain length and functional group. ${ }^{3}$ Having a coating with simultaneously more than one function thus represents a challenge for silane based molecules,

\footnotetext{
anstitute of Materials Science and Nanotechnology, Bilkent University, 06800 Ankara, Turkey

${ }^{b}$ UNAM-National Nanotechnology Research Center, Bilkent University, 06800 Ankara, Turkey

'Department of Physics, Bilkent University, 06800 Ankara, Turkey. E-mail: bayindir@ nano.org.tr

$\dagger$ Electronic supplementary information (ESI) available: Experimental procedures, XPS, contact angle measurement, AFM, and ellipsometry and confocal microscopy data. See DOI: $10.1039 / \mathrm{c} 4$ tb01283f

$\ddagger$ Equal contribution to the research.
}

since an alteration in one directly affects the nature of whole coating. Coating with more than one molecule is hypothetically possible, either simultaneously or sequentially. However, it is not practical to perform in every case due to easy polymerization and unpredictable assembly behavior of silanes. ${ }^{1}$

Here we propose an alternative strategy for realizing antifouling surfaces with bioconjugation capability by using only a single molecule for surface modification. We utilized THPMP, a commercial small silane-based molecule, to produce a bioconjugable and anti-fouling silica surface (see ESI $\dagger$ for details). Phosphonates have been used as functional groups stabilizing silica containing nanoparticles against aggregation ${ }^{4}$ or sites for covalently anchoring primary amines to other molecules such as nucleic acids. ${ }^{5}$ Also, it has been shown previously that methylphosphonate containing silica nanoparticles exhibited significantly reduced non-specific biological interactions, ${ }^{6}$ and our research group has recently demonstrated that methylphosphonate conjugation particularly decreased protein adsorption on silica nanoparticles. ${ }^{7}$ However, to the best of our knowledge, neither the characteristics of these phosphonatebased films were investigated in detail, nor were their bioconjugation properties utilized together with their protein resistant characteristics. In this study, we experimentally characterized the THPMP film, and examined it regarding its protein resistance and bioconjugation characteristics. Our results show that THPMP could effectively be used for producing simultaneously bioconjugable and protein resistant silica surfaces in a facile manner, as shown in Scheme 1.

After coating the silica surfaces with THPMP by a facile method that we developed (see ESI $\dagger$ for details), we conducted XPS, contact angle, AFM and ellipsometry analyses to characterize the coating. Fig. 1 represents the comparison of high resolution XPS scans of P2p, Si2p, O1s and C1s regions for bare and THPMP coated silica surfaces. As also could be seen from the survey spectra (Fig. S1, ESI $\dagger$ ), the P2p signal at $133.2 \mathrm{eV}$ only arises from the THPMP coated sample, showing existence of $P$ over the surface. Due to the fact that low abundance of $P$ on the surface, the P2p signal is much lower compared to that other 

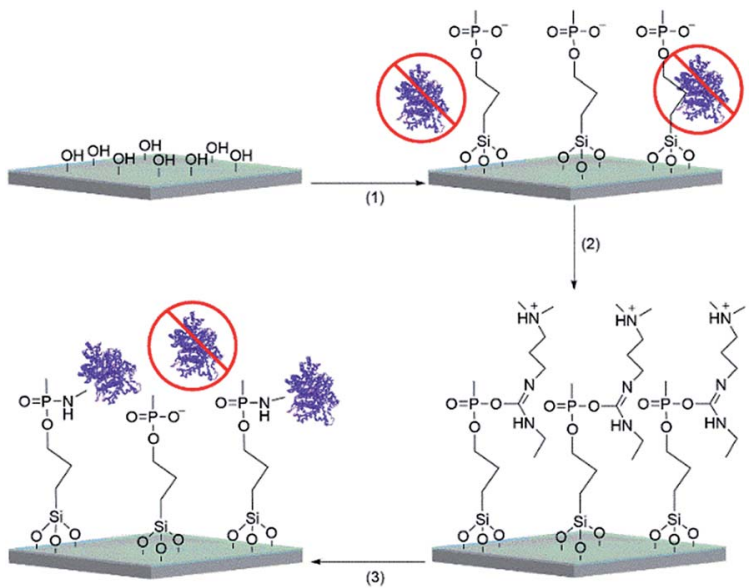

Scheme 1 Piranha treated silica surface is first coated with THPMP (1), which forms a protein resistant thin film. Methylphosphonates could be activated temporarily by using a cross-linker (2). After the covalent attachment of proteins via their primary amines (3), the coating reverts to its bio-inert state.
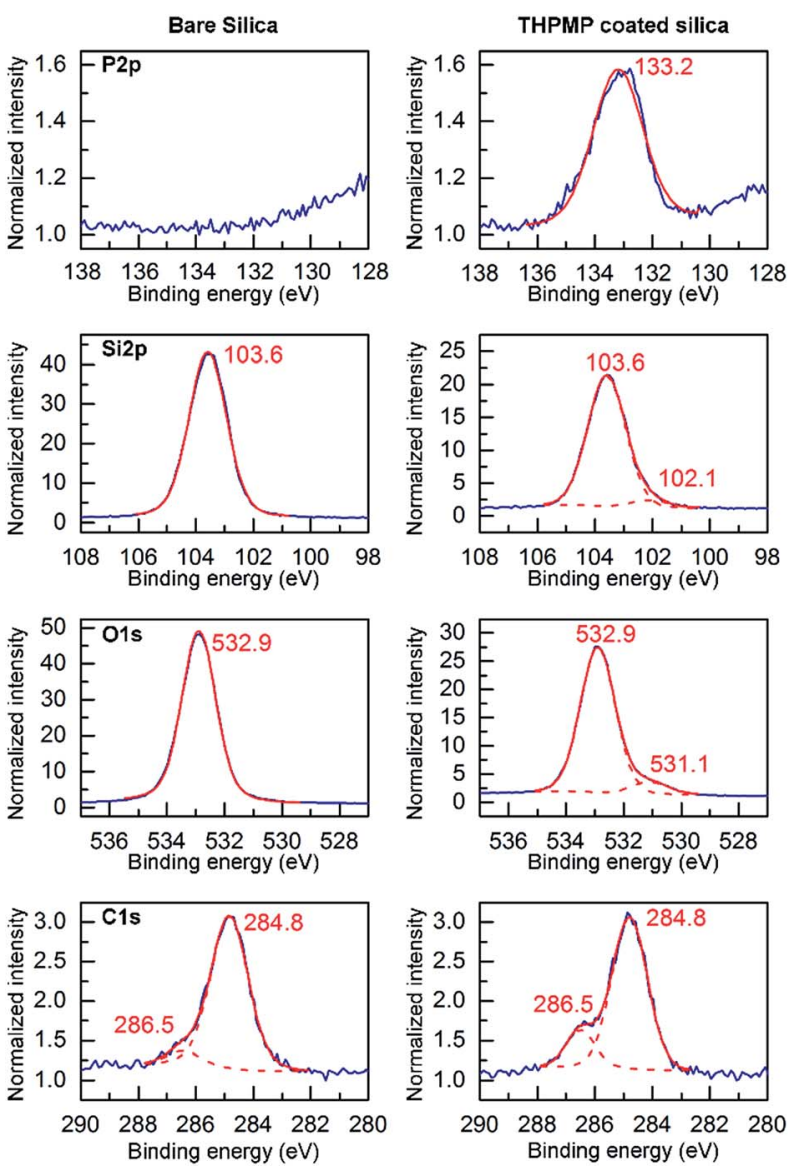

Fig. 1 Comparison of high resolution XPS scans of P2p, Si2p, O1s and $\mathrm{C} 1 \mathrm{~s}$ regions for the bare silica and THPMP coated silica surfaces. Original data are given in blue lines, while fits for single peaks and envelopes are given in dashed and solid red lines, respectively. elements have (Table S1, ESI $\dagger$ ). The XPS spectrum of bare silica is known to possess single peaks for both silicon and oxygen, while functional group related peaks emerge after organosilane modification, ${ }^{8}$ which is coincident with our data. As seen in the Si2p scan of the bare silica surface, a single peak at $103.6 \mathrm{eV}$ is from an oxygen-rich Si binding environment. ${ }^{9}$ The THPMP coated silica shows an additional peak, located at $102.1 \mathrm{eV}$, suggesting a $\mathrm{C}-\mathrm{Si}-\mathrm{O}$ bonding. ${ }^{10}$ This peak with lower energy verifies that a slightly carbon-rich environment is induced on the Si surface due to THPMP bonding. Additionally, O1s scans of the bare and THPMP coated silica surfaces show a common peak located at $532.9 \mathrm{eV}$. This peak arises from thermal oxide. ${ }^{11}$ After the THPMP coating, a lower energy peak located at 531.1 $\mathrm{eV}$ appeared. This peak shows $\mathrm{Si}-\mathrm{O}$ bonding $^{8}$ between THPMP molecules and the silica surface. C1s scans of the bare and THPMP coated silica surfaces show two common peaks at 284.8 and $286.5 \mathrm{eV}$, which are attributed to $\mathrm{C}-\mathrm{C}$ bonding and $\mathrm{C}-\mathrm{O}$ bonding, ${ }^{12}$ respectively. The intensity of the lower energy peak at $286.5 \mathrm{eV}$ increases significantly after the THPMP coating due to covalent bonding of the THPMP molecules over the silica surface.

Static contact angle measurement of a piranha cleaned surface revealed a contact angle of $\sim 3^{\circ}$, since the surface became highly hydrophilic after the piranha treatment (Fig. S2, ESI $\uparrow$ ). After the THPMP coating, the surface gained a static contact angle of $49.4 \pm 2.0^{\circ}$ (Fig. S2b, ESI $\dagger$ ) indicating the existence of a moderately hydrophilic methylphosphonate containing thin film over the silica surface. Additionally, in another study it was indicated that a contact angle of $\sim 50^{\circ}$ had been observed at hydroxyl terminated and compact monolayers of phosphonate coatings. ${ }^{13}$

We did AFM measurements to gain information regarding the morphology of the THPMP coating. AFM results (Fig. S3, ESI $\dagger$ ) demonstrate a roughened surface after the coating (Fig. S4, ESI†). Also, this has been commonly observed in other silane based coatings. ${ }^{14}$ Rms surface roughnesses from $1 \times 1$ $\mu \mathrm{m}$ scanned areas of the bare and THPMP coated silica surfaces were calculated to be $0.59 \pm 0.03 \mathrm{~nm}$ and $2.08 \pm 0.09 \mathrm{~nm}$, respectively.

Additionally, from ellipsometry measurements, we assessed the thickness of the THPMP film as $0.65 \pm 0.02 \mathrm{~nm}$ (Table S2, ESI $\dagger$ ). This estimated thickness is less than the rms values we obtained; however, since the ellipsometry measurement is performed over a much larger area compared to AFM, we observe an average characteristic of the THPMP film. Rms values higher than the thickness were previously reported, which occur due to local polymerization of organosilane molecules over the surface. ${ }^{14}$ Thus, we suggest that our results both from AFM and ellipsometry do not contradict with each other. Our results indicate that we coated the silica surface completely with a thin THPMP film with partial aggregations.

We tested the protein resistance of THPMP coating against four different proteins: BSA, lysozyme, fibrinogen and $\gamma$-globulin. BSA and $\gamma$-globulin are serum proteins with different molecular weights, while fibrinogen, another serum protein, is used in adsorption studies due to its large molecular weight and sticky nature. Lysozyme, on the other hand, a small protein 
which is positively charged under our experimental conditions (at pH 7.4), is used to observe whether any electrostatic effects are present. ${ }^{15}$ We did XPS measurements to characterize the adsorption of these proteins. High resolution XPS scans of N1s regions of piranha treated and THPMP coated silica surfaces were compared after being exposed separately to the aforementioned four protein solutions.

The results shown in Fig. 2 represent that N1s signals significantly decreased from the samples having THPMP coating. While comparing the peak intensities quantitatively, instead of directly using atomic percentages of the survey, ${ }^{16}$ we performed a background correction, because we observed a significant difference in the baselines among piranha treated and THPMP coated silica surfaces, probably caused by the increased surface roughness. We also performed ellipsometry measurements to compare BSA films formed over silica surfaces before and after the THPMP coating (Fig. S5, ESI†). The amount of BSA adsorbed is estimated to reduce fivefold according to the measured film thicknesses, ${ }^{17}$ which is comparable to similar ellipsometry measurements. ${ }^{18}$ It is important here to note that it is difficult to assess the actual thickness solely depending on ellipsometry, especially when the surface contains voids, ${ }^{19}$ as in the protein adsorption case. Nevertheless, the protein thicknesses we deduced from the ellipsometric fits from different spots on the wafer (Table S2, ESI†) demonstrate that there is a significant qualitative difference between piranha treated and THPMP coated silica surfaces, coinciding with our XPS results.

Many factors are shown to contribute to protein resistance, among which the most prominent ones are hydrophilicity, inclusion of hydrogen-bond acceptors but not donors, and neutral overall charge. ${ }^{20}$ The methylphosphonate group is hydrophilic, contains two oxygens as hydrogen-bond acceptors but no hydrogen-bond donors, and is neutral or very slightly negatively charged in PBS (pH 7.4), as we measured previously. ${ }^{7}$ Therefore, since the characteristics of the THPMP molecule fit within the empirically described domain of high protein

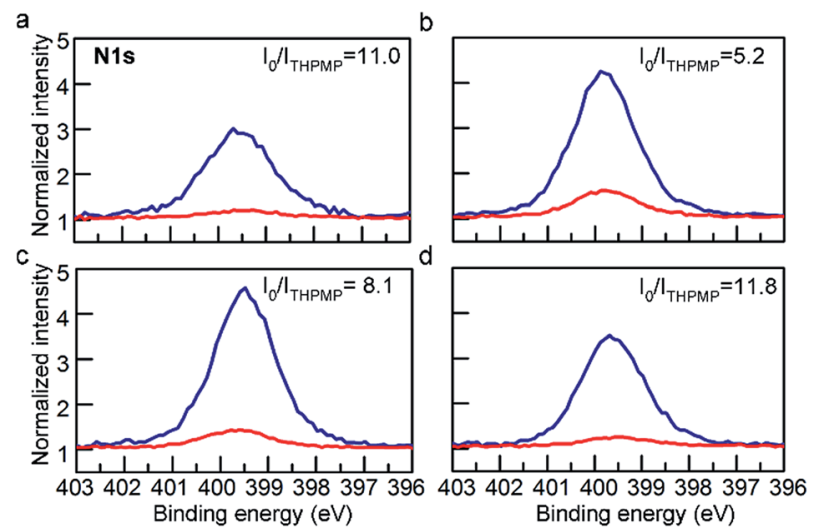

Fig. 2 High resolution XPS scans of N1s of piranha treated and THPMP coated silica surfaces after exposure to (a) BSA, (B) $\gamma$-globulin, (c) fibrinogen, and (d) lysozyme solutions. THPMP coating showed significant resistance to all tested proteins. The ratios of intensities of piranha treated $\left(I_{0}\right)$ and THPMP coated $\left(I_{\text {THPMP }}\right)$ surfaces were calculated after background correction. resistance, it could be anticipated that THPMP coating over silica would show a considerable protein resistance.

In order to covalently conjugate proteins to the methylphosphonate groups, we used EDC as an activator (see ESI $\dagger$ for details). EDC attacks the negatively charged terminal oxygen of methylphosphonate, forming an unstable $O$-acylisourea intermediate, which is then replaced by a primary amine. As a verification, we applied EDC/NHS chemistry (see ESI $\dagger$ for details) and performed a high resolution N1s scan to observe NHS esters (Fig. S6, ESI $\dagger$ ). We observe two distinct peaks at 400.0 and 402.2 $\mathrm{eV}$, corresponding to $\mathrm{N}-\mathrm{C}$ and $\mathrm{N}-\mathrm{O}$, respectively, indicating the formation of NHS ester termination. ${ }^{21}$ In a previous study regarding EDC activation of methylphosphonate for covalent attachment of primary amines, phosphoramidate chemistry was suggested; i.e., the unstable $O$-acylisourea was suggested to be formed via the methyl group of methylphosphonate. ${ }^{22}$ However, according to our XPS data, we observe the typical N-C and $\mathrm{N}-\mathrm{O}$ bonding verifying the formation of NHS esters. Our results imply that the NHS ester formation occurs via negatively charged oxygen terminal of THPMP molecules, suggesting a phosphonamidate structure. ${ }^{23}$

We utilized confocal microscopy to examine protein resistance and bioconjugation simultaneously and compare different samples quantitatively, using FITC labeled BSA (Fig. 3). First, we verified that the THPMP coated surface is protein resistant, as could be seen from the confocal images of the piranha treated (Fig. 3a) and THPMP coated (Fig. 3b) quartz wafers, comparing their fluorescence intensities. The protein resistance for longer periods was also investigated separately (Fig. S7, ESI $\dagger$ ). Then, we covalently attached FITC-BSA over the THPMP coated quartz wafers after EDC activation (Fig. 3c), and observed a significant fluorescence, the intensity of which is comparable to the nonspecific adsorption. Normally, it is difficult to distinguish covalent coupling from nonspecific adsorption. However, we assert that covalent conjugation occurred, since proteins were successfully accommodated over the THPMP film after EDC activation, which was otherwise protein resistant.

We also tested the recovery of THPMP after EDC activation by washing the wafer thoroughly with ultrapure water prior to incubation with FITC-BSA. We observed a significant loss in the fluorescence (Fig. 3d), indicating the reversibility of the EDC activation. High resolution XPS scans of P2p and N1s regions of recovered THPMP coatings on thermal silica (Fig. S8, ESI $\dagger$ ) also verified that the film chemically returns to its unmodified form. The quantitative comparison of all fluorescence intensities is also given (Fig. 3e). The results show that THPMP possesses a dramatic protein resistant behavior. Additionally, covalent attachment of proteins is feasible by activating the THPMP coated surface with EDC, which is totally reversible in terms of protein resistance. The fact that $O$-acylisourea is quite unstable in aqueous environments could be advantageous, regarding the recovery of protein resistance properties of the THPMP film after EDC activation and covalent modification. As a further control, we covalently conjugated amine modified ssDNA to the wafers after EDC activation, and still observed significant protein resistance after bioconjugation (Fig. S9, ESI $\dagger$ ). 

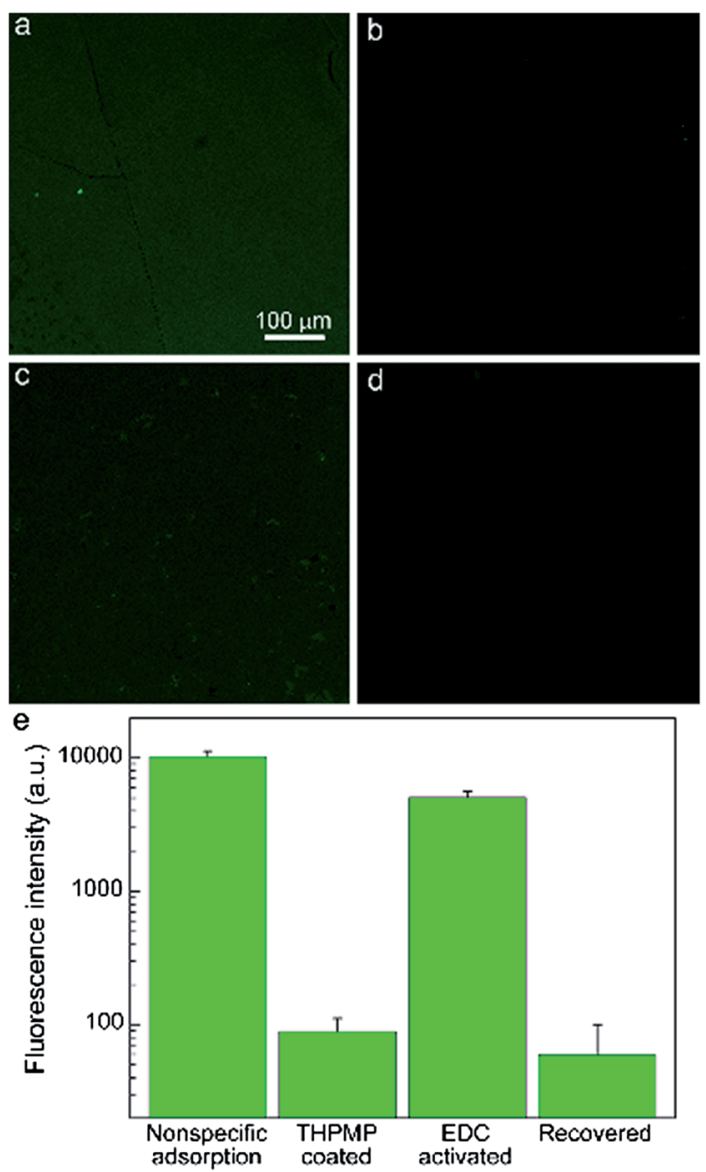

Fig. 3 Nonspecific adsorption of FITC-BSA over the piranha treated quartz surface (a) is almost diminished after THPMP coating (b). Covalent attachment, which produces a comparable signal level to nonspecific adsorption, is possible by EDC activation (c), while THPMP coating can be reverted to its protein resistant original state later (d). The comparison of the fluorescence intensities (e) enables quantitative comparison of the amount of protein present over the surface.

Confocal microscopy measures emitted photons from fluorophores attached to the proteins, which is proportional to the amount of proteins on the surface, suggesting that THPMP induces around two orders of magnitude reduction in the amount of adsorbed BSA. According to the XPS measurements, the resistance towards other proteins is expected to be on the same order as well. Although not coinciding with the obtained XPS and ellipsometry results, when the limitations of these research tools are considered, such as difficulty of measurement on rough surfaces with XPS, ${ }^{24}$ or thin film approximation of the ellipsometer, ${ }^{17}$ we could assume that the confocal microscopy measurements are dependable for a quantitative comparison.

AFM images were taken after covalent coupling of BSA over the THPMP coated silica surface with two different scanned areas (Fig. S10 and 11, ESI $\dagger$ ). Rms surface roughnesses of scanned wide and narrow areas from the covalently BSA conjugated surface were measured to be $3.82 \pm 0.16 \mathrm{~nm}$ and $3.62 \pm 0.56 \mathrm{~nm}$, respectively. The surface roughness increased after covalent coupling of BSA to the THPMP film. Moreover, the surface shows a uniform distribution of BSA over a wide area range. It can be seen from the figure that the proteins formed a layer following the pattern of the THPMP coating. We also took an AFM image of non-specifically bound BSA onto a piranha cleaned silica surface with $1 \times 1 \mu \mathrm{m}$ scanned area (Fig. S12, ESI $\dagger$ ). The Rms surface roughness was measured to be $1.23 \pm$ $0.19 \mathrm{~nm}$ and a significantly different morphology from that of the BSA conjugated THPMP film was observed.

In conclusion, we demonstrate that methylphosphonate containing organosilanes could effectively be used as protein resistant coatings which are simultaneously bioconjugable. Both coating of silica with methylphosphonate and covalent protein conjugation are quite straightforward and efficient, and activation of methylphosphonates could easily be reverted, forming an inert coating around the bioconjugated moiety. Using only a single molecule in a multifunctional manner provides an unprecedented flexibility to the model we propose, which in our opinion could find important applications especially in biomedical fields, such as smart biocompatible implants, targeted drug delivery, and high selectivity and sensitivity biosensors.

\section{Acknowledgements}

We thank Adem Yildirim for valuable discussions during the design of the study and interpretation of the experimental results. This work was supported by the TUBITAK Grant no. 112T612. M.B. acknowledges partial support from the Turkish Academy of Sciences (TUBA).

\section{Notes and references}

1 M. J. Banuls, R. Puchades and A. Maquieira, Anal. Chim. Acta, 2013, 777, 1 .

2 M. Malmsten, K. Emoto and J. M. Van Alstine, J. Colloid Interface Sci., 1998, 202, 507.

3 S. Herrwerth, W. Eck, S. Reinhardt and M. Grunze, J. Am. Chem. Soc., 2003, 125, 9359.

4 D. Gerion, F. Pinaud, S. C. Williams, W. J. Parak, D. Zanchet, S. Weiss and A. P. Alivisatos, J. Phys. Chem. B, 2001, 105, 8861.

5 J. T. G. Pena, C. Sohn-Lee, S. H. Rouhanifard, J. Ludwig, M. Hafner, A. Mihailovic, C. Lim, D. Holoch, P. Berninger, M. Zavolan and T. Tuschl, Nat. Methods, 2009, 6, 139.

6 C. D. Medley, S. Bamrungsap, W. H. Tan and J. E. Smith, Anal. Chem., 2011, 83, 727.

7 A. Yildirim, E. Ozgur and M. Bayindir, J. Mater. Chem. B, 2013, 1, 1909.

8 R. A. Shircliff, P. Stradins, H. Moutinho, J. Fennell, M. L. Ghirardi, S. W. Cowley, H. M. Branz and I. T. Martin, Langmuir, 2013, 29, 4057.

9 P. H. T. Ngamou, J. P. Overbeek, H. M. van Veen, J. F. Vente, P. F. Cuperus and M. Creatore, RSC Adv., 2013, 3, 14241.

10 A. Afzal, H. M. Siddiqi, S. Saeed and Z. Ahmad, RSC Adv., 2013, 3, 3885. 
11 X. N. Xie, M. Deng, H. Xu, S. W. Yang, D. C. Qi, X. Y. Gao, H. J. Chung, C. H. Sow, V. B. C. Tan and A. T. S. Wee, J. Am. Chem. Soc., 2006, 128, 2738.

12 L. H. Wang, Y. Tian, H. Y. Ding and J. D. Li, Eur. Polym. J., 2006, 42, 2921.

13 A. Cattani-Scholz, D. Pedone, M. Dubey, S. Neppl, B. Nickel, P. Feulner, J. Schwartz, G. Abstreiter and M. Tornow, ACS Nano, 2008, 2, 1653.

14 J. A. Howarter and J. P. Youngblood, Langmuir, 2006, 22, 11142.

15 R. G. Chapman, E. Ostuni, S. Takayama, R. E. Holmlin, L. Yan and G. M. Whitesides, J. Am. Chem. Soc., 2000, 122, 8303.

16 J. H. Lee, J. Kopecek and J. D. Andrade, J. Biomed. Mater. Res., 1989, 23, 351.
17 M. Stenberg and H. Nygren, J. Phys., Colloq., 1983, 44, C10. 18 M. Q. Zhang, T. Desai and M. Ferrari, Biomaterials, 1998, 19, 953.

19 P. Tengvall, I. Lundstrom and B. Liedberg, Biomaterials, 1998, 19, 407.

20 E. Ostuni, R. G. Chapman, R. E. Holmlin, S. Takayama and G. M. Whitesides, Langmuir, 2001, 17, 5605.

21 Y. Coffinier, N. Nguyen, H. Drobecq, O. Melnyk, V. Thomy and R. Boukherroub, Analyst, 2012, 137, 5527.

22 A. Popat, J. Liu, G. Q. Lu and S. Z. Qiao, J. Mater. Chem., 2012, 22, 11173.

23 I. Wilkening, G. del Signore and C. P. R. Hackenberger, Chem. Commun., 2011, 47, 349.

24 B. A. Kobe, S. Ramamurthy, M. C. Biesinger, N. S. McIntyre and A. M. Brennenstuhl, Surf. Interface Anal., 2005, 37, 478. 